\title{
A Preliminary Study on Edge Computing
}

\author{
Da Xu, Jinting Bai \\ National Meteorological Information Center, 46, Zhonguancun North Road, Beijing 46, China
}

\begin{abstract}
Edge computing is a distributed computing architecture in which the operations of applications, data and services move from the central node to logical edge nodes of the network. This paper first summarizes the development history of edge computing and the evolution process from content delivery network to cloud computing, fog computing and edge computing, and analyzes the related achievements in various industries; then introduces two popular edge computing frameworks; and finally proposes an assumption of information system architecture based on edge computing, and discusses the problems and challenges that edge computing will solve and face in modern meteorological service.
\end{abstract}

Keywords: Edge computing, Architecture, Meteorological service.

\section{Introduction}

With the constant modernization and information construction of China's meteorological industry, and the completion of the meteorological big data cloud platform ("Tianqing") and the real-time monitoring system of integrated meteorological services ("The Mirror")[14], the information and data have been increasing exponentially. In this context, effectively processing and utilizing mass data and improving service quality has become the primary goal of system optimization. In the construction process of Tianqing and The Mirror, the concept of cloud computing was introduced. Cloud computing essentially originates from super-large-scale distributed computing, which realizes the information exchange and storage between nodes through the network. The focus of cloud computing is to meet different service needs through resource recombination. In the actual meteorological service scenarios, the data center (cloud server) and the observation device (terminal) are too far away from each other, which may cause problems such as latency, bandwidth and energy consumption. As a result, a new solution is needed to make up for the deficiency of cloud computing. Edge computing is a computing model that processes and stores data at the edge of the network, as close to the user terminal equipment, so as to provide reliable and stable services for users nearby. Due to the short distance between the edge server and the terminal equipment, the edge server can directly provide services to reduce the latency, and transmit the calculated indicators to the data center, thus relieving the bandwidth pressure and reducing the server energy consumption of the data center to a certain extent. At present, the investment in high technology in China mainly focuses on $5 \mathrm{G}$ and $\mathrm{AI}$, while the edge computing architecture possesses an absolute advantage in the Internet of Thing[1] and there are more and more studies on edge computing. Since 2015, the studies about edge computing has been exploded, in both theory and application. In this paper, Section 2 introduces the development history and current situation of edge computing; Section 3 details two popular edge computing frameworks and their design features; Section 4 proposes an assumption of an edge computing information system architecture based on meteorological observation network, and presents a vision for the future.

\section{Rise of Edge Computing}

\subsection{CDN}

Edge computing can be traced back to the content delivery network (CDN), which was put forward by Akamai Company in 1998. CDN is an Internet-based cache network. Relying on the cache servers deployed in different locations, CDN can direct users' access to the nearest cache server through the load balancing, content delivery, scheduling and other functional modules of the central platform, so as to reduce network congestion and improve the response speed and hit rate of users' access. CDN emphasizes data backup and cache, while the basic idea behind edge computing is function cache, which is data processing.

\subsection{Cloud Computing}

In August 2006, Eric Schmidt, CEO of Google, pointed out that "there is now a new business model which is on the premise that the architecture of data services should be operated on a server called 'cloud computing', that is, in a cloud. You can access these data, as long as there is a suitable browser or access method, regardless of the equipment." Cloud computing is named by Google, but a few weeks after Eric Schmidt put forward the term "cloud computing", Amazon launched the Elastic Compute Cloud (EC2), which included the term "cloud".

\subsection{Fog Computing}

The concept of fog computing was first proposed by Cisco in 2011. According to Cisco, fog computing is comprised of more decentralized functional computers with weaker performance. IoT technologies seeps into our daily life. Fog computing is a semi-virtualized service computing architecture model between cloud computing and personal terminal devices, and fog servers have the functions of storage, computing and communication. Fog computing makes full use of the computing power of each single node and provides services for users through wireless transmission. Compared with cloud computing, fog computing features low latency, location awareness, wide geographical distribution and adaptability to mobility, and supports more edge nodes. The 
architecture of fog computing is more distributed and closer to the edge of the network, which facilitates the deployment of mobile services and enables a wider range of node access.

\subsection{Edge Computing}

Edge computing saw a golden period of development from 2015 to 2017[1-4]. It has attracted close attention from the academic and industrial circles at home and abroad, because it can meet the needs of the Internet of Everything. In May 2016, the National Science Foundation (NSF) took edge computing as a prominent field, in lieu of cloud computing in a computer system study. The team of Professor Shi Weisong from Wayne State University gave a formal definition of edge computing: edge computing is a new computing model that performs computing at the edge of network[1]. The objects of edge computing include the downlink data from cloud services and the uplink data from the Internet of Everything services, and "edge" refers to the computing and network resources between data sources and cloud computing center paths. Shi Weisong team also published an article titled "Edge Computing: Vision and Challenges" about the development and challenges of edge computing, elaborating the programming feasibility, naming, data abstraction, service management, privacy security and optimization[4]. In October 2016, NSF Workshop on Grand Challenges in Edge Computing was held, which focused on the following three topics: the development goal of edge computing in the next 5-10 years, the challenges that may face in the process of achieving the goal, and the cooperation and collaboration among academia, industry and government to deal with the challenges. In addition, ACM/IEEE Symposium on Edge Computing (SEC) was jointly held by ACM and EEE. This is the first academic conference on edge computing in the world. In September 2015, the European Telecommunications Standards Institute (ETSI) published a white paper on mobile edge computing, and in March 2017, ETSI officially changed the name of its industry specification group for mobile edge computing into Multi-access Edge Computing (MEC) Industry Specification Group, aiming at better meeting the application requirements of edge computing and formulating relevant standards. In November 2015, Cisco, ARM, Dell, Intel, Microsoft and Princeton University jointly established OpenFog Consortium which is mainly committed to construction of Fog Reference Architecture. In order to promote the combination with application scenarios at the edge, OpenFog Consortium was merged into the Industrial Internet Consortium in December 2018.

The development of China's edge computing almost keeps in pace with that of global edge computing, especially in the field of intelligent manufacturing. In November 2016, the Edge Computing Consortium was jointly established by Huawei Technologies Co., Ltd., Shenyang Institute of Automation under the Chinese Academy of Sciences, China Academy of Information and Communications Technology, Intel and ARM, with an aim to promote the "government-industry-university-research" cooperation and lead the healthy and sustainable development of the edge computing industry. In September 2018, WAIC Forum was held in Shanghai on the theme of "Edge Computing, Smart Future".

\section{Edge Computing Architecture}

\subsection{EdgeX Foundry}

EdgeX[5] Foundry is an open-source platform hosted by Linux Foundation, providing a common framework for IoT (Internet of Things) edge computing. The core of EdgeX Foundry is an interoperability framework, which is designed to extend the platform with plug-and-play components and provide a set of IoT deployment scheme, regardless of hardware and operating system.

EdgeX Foundry, based on a loosely coupled microservice architecture, is mainly divided into four layers (device services layer, core services layer, supporting services layer and application services layer), which are targeted at the services in the north and south ends. As shown in Figure 1, the device services layer is mainly targeted at the IoT devices and sensors in the south end, supports multiple device interface connections, and transmits data into the core services layer by converting standard protocols and proprietary protocols into general APIs; the application services layer is targeted at the cloud computing services in the north end through standard protocols.

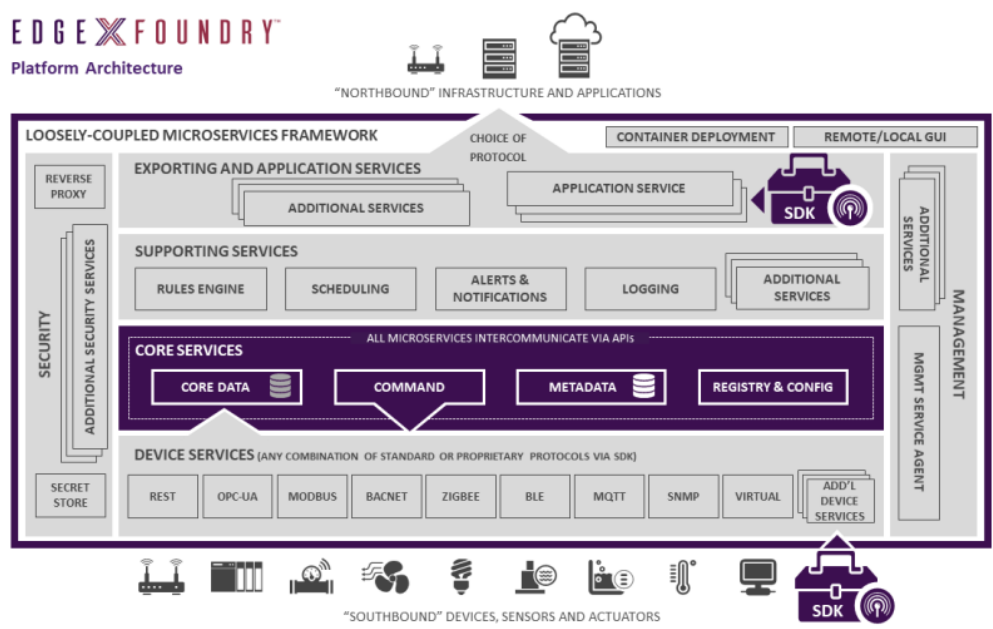

Figure 1: EdgeX Foundry Platform Architecture 
Main features of EdgeX Foundry:

1) There is no need for users to pay attention to the differences among underlying hardware CPU (e.g., x86 and ARM), operating system (OS) (e.g., Linux, Windows and Mac OS) and application environment (e.g., Java, JavaScript, Python, Go Lang and $\mathrm{C} / \mathrm{C}++)$.

2) Enable flexible management of services according to the computing power of devices.

3) Allow computing functions to be distributed among multiple edge hardware nodes or multiple processors in a given node.

4) Meet the requirements of industrial safety, manageability, performance and reliability, and maintain high extendibility.

5) Directly replace a microservice component with a higher-performance version by virtue of the microservice characteristics, without changing the architecture.

\subsection{BAETYL2.0}

Baetyl2.0 (pronounced as "Beetle")[6] provides a common platform for edge computing, which integrates different types of hardware facilities and device functions into a standardized container runtime environment and API, so that applications, services and data flows can be efficiently managed through the remote console whether in the cloud or locally.

Baety2.0 is mainly composed of two parts: cloud management suite and edge computing framework. Specifically, the setup of intelligent edge core devices, identity making, rule and policy making, function writing and AI modeling are conducted in the cloud, and then configuration files and execution files are generated and distributed to the local running package through terminal-cloud collaboration; data collection, message distribution, function calculation and AI inference are completed in the local running package near the edge device. Through one-click release and non-inductive deployment, Baety2.0 greatly improves the speed of intelligent iteration, and achieves the goal of "training, management and configuration in the cloud, and collection, transmission, computing and inference locally".

In terms of architecture design, Baetyl2.0 adopts container-based design, and relying on the cross-platform support features of Kubernetes container, guarantees the environmental consistency and standardization of intellectual edge in different systems and platforms. In addition, the framework isolates and limits the container resources, accurately allocates CPU, memory and other resources of each running instance, and improves the resource utilization efficiency.

As shown in Figure 2:

\section{Kubernetes Mode}

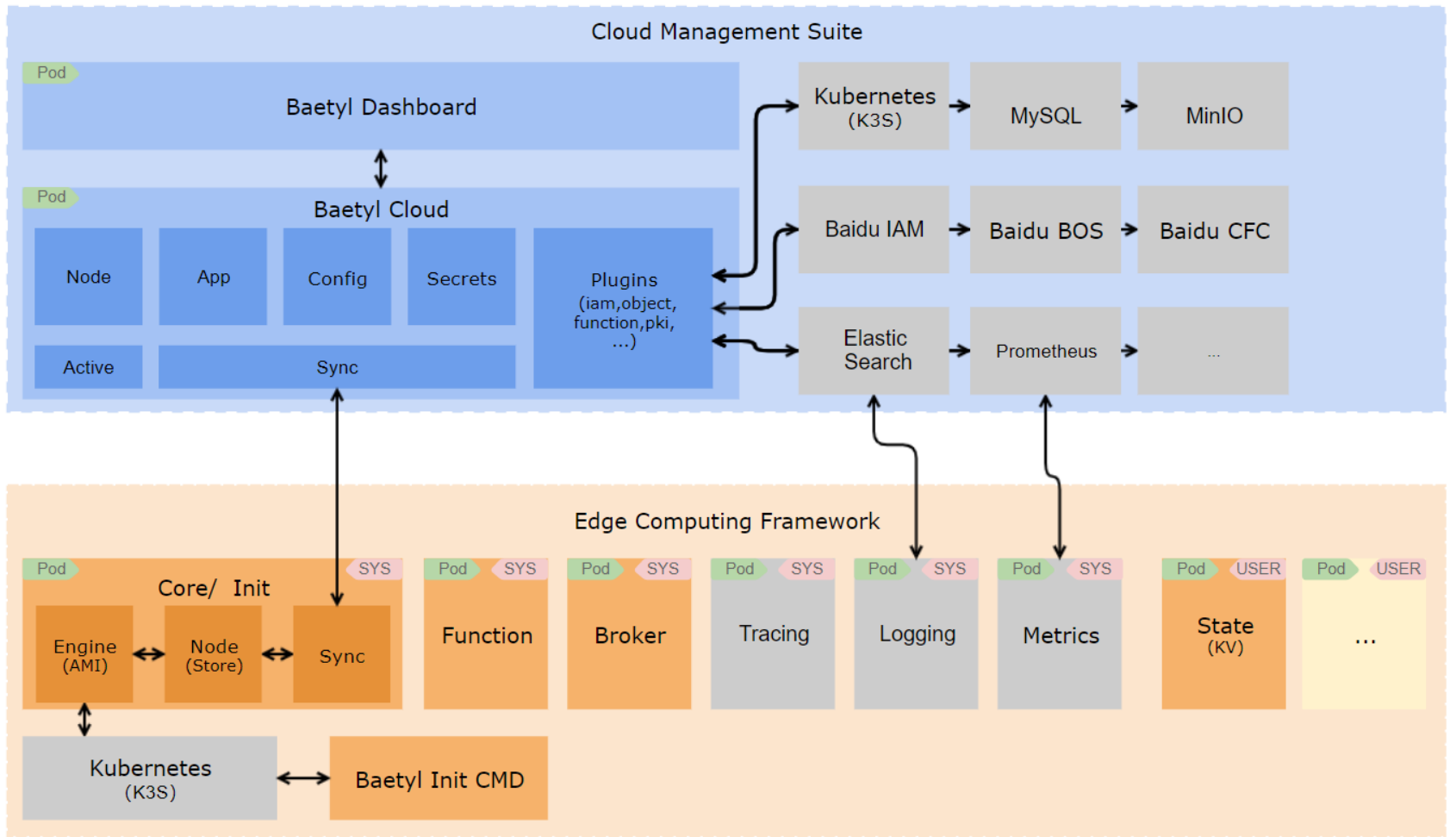

\begin{tabular}{c|c|c|}
$\begin{array}{c}\text { Baetyl } \\
\text { Cloud }\end{array}$ & Baetyl & Third \\
Modules & Edge & Part \\
Modules & Modules
\end{tabular}

App runs in K8S Pod

App runs in system namespace, app is configurated by baetyl system

App runs in user namespace, app is configurated by user

Figure 2: BAETYL2.0 Architecture 
The Cloud Management Suite manages all resources, including nodes, applications, configuration, deployment, etc. All functions are realized by means of plugins, which facilitates function expansion and access to third-party services, and provides rich applications.

The Edge Computing Framework runs on Kubernetes at the edge node, manages and deploys all node applications, and provides various capabilities through application services.

Main functional modules:

1) Baetyl-init: responsible for activating the edge node to the cloud and initializing baetyl-core, and will exit after all tasks are completed.

2) Baetyl-core: responsible for local node management (node), data synchronization with cloud (sync) and application deployment (engine).

3) Baetyl-function: the proxy for all function runtime services.

\section{The Application of Edge Computing in Meteorology}

\subsection{Application Background}

With the continuous development of automatic and information-based meteorological observation technologies in China, the observation has realized full coverage of space-based, land-based, air-based and sea-based systems. In April 2020, China's ground-based observation stations realized automatic monitoring. The future meteorological information system entails a land-sea-air-space integrated multi-dimensional observation system to monitor the global situations in real time, and facilitate the commands and decisions about weather, climate, and early warning for disaster prevention and mitigation. The diversity of observation equipment and systems requires the meteorological transmission system (CTS), meteorological big data cloud platform ("Tianqing") and integrated meteorological service monitoring system ("The Mirror") to have fast information exchange ability and powerful computing ability, and to complete the integration, processing and analysis of massive data at each observation station with low latency. Characterized by high security and low service latency, edge computing provides a new solution for the future meteorological information system architecture.

\subsection{Application Characteristics}

The application of edge computing in meteorology faces a more complex network environment, compared with its application in other fields, which brings forward higher demand for the agility, flexibility and robustness of the information system. The characteristics of meteorological industry and other industries are compared in Table 1 below:
Table 1: Comparison of the Application Characteristics of Edge Computing

\begin{tabular}{ccc}
\hline Features & Meteorology & Other fields \\
\hline Purpose & Accurate detection and & Reduce the service \\
Confidentiality & Obsecast, and fine service & response time \\
Timeliness & High & Low \\
Robustness & 24h uninterrupted operation & High \\
Network & MPLS VPN & Average \\
Hardware & Autonomous and controllable & Internet \\
\hline
\end{tabular}

\subsection{Architecture Design}

Edge computing suppose strong computing power and low service latency through widely distributed edge nodes and computing sink. In meteorological transmission, edge computing can reduce the transmission of original data, cut down the bandwidth and improve the index computing power. Based on the calculation characteristics of whole-process meteorological data, this paper proposes a "cloud-edge-terminal" integrated reference architecture design of information system based on edge computing to optimize the existing whole-process computing framework, as shown in Figure 3.

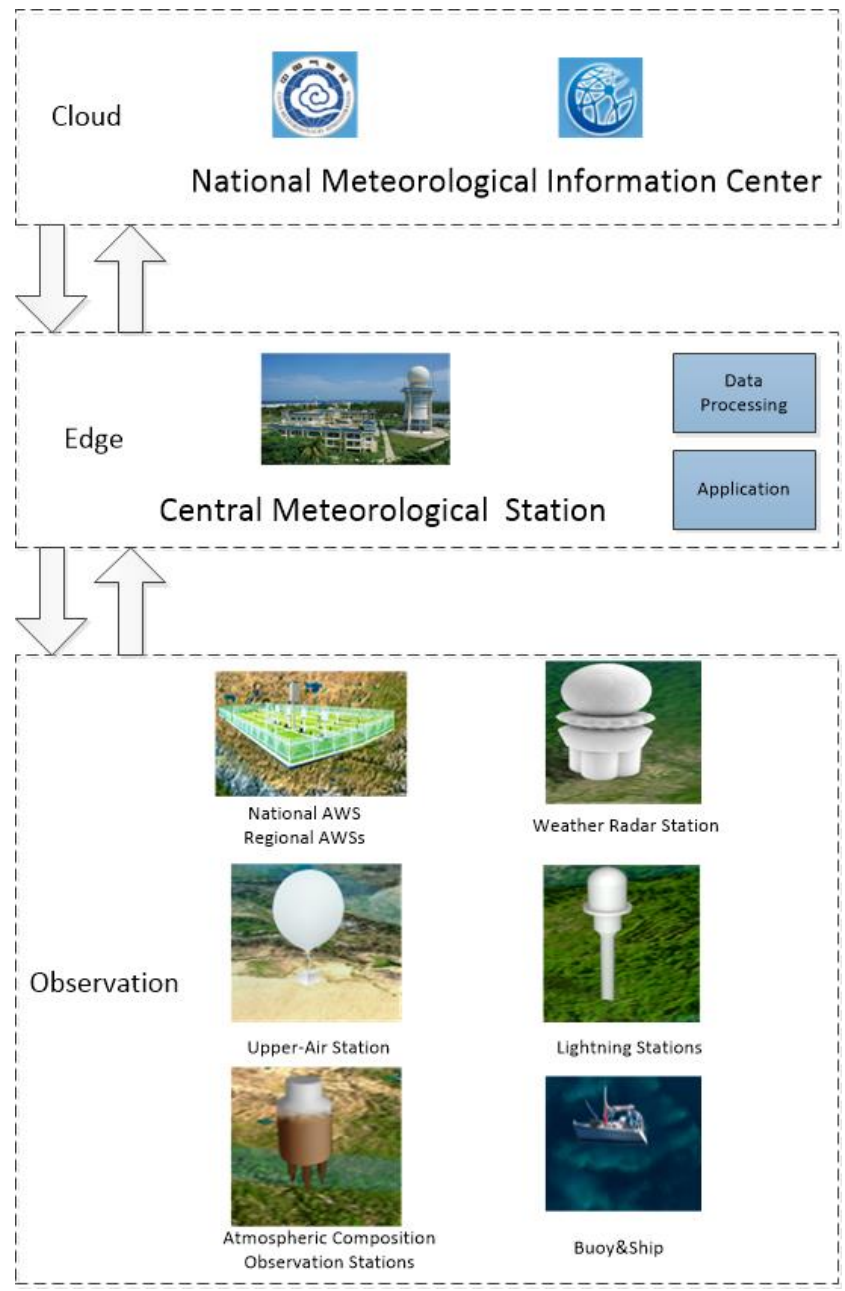

Figure 3: Meteorological System Architecture Based on Edge Computing

In this architecture, all meteorological observation devices are located in the terminal layer, and the meteorological stations in the edge layer are equipped with the additional functions of computing processing and application management. 
1) Computing processing: At present, the whole-process computing framework of meteorological data runs in the cloud, which is National Meteorological Information Center. The structure of the computing framework is shown in Figure 4.

After the original data enters the gateway, the preprocessing module enriches the information (supplementing station information, latitude \& longitude, etc.), generates intermediate result information, and uses Spark to get the final results through big data computing. With the increase of meteorological data types and observation stations, the existing computing framework has been found to have insufficient computing capacity. Under the "cloud-edge-terminal" architecture, the preprocessing module can sink to the edge layer-meteorological center station, to process the original data in advance, and then the processed intermediate results can be uploaded to the cloud for final aggregation computing, which can greatly reduce the computing and storage pressure in the cloud and improve the computing efficiency. Meanwhile, the data format check function can sink to the edge layer to reduce the gateway load.
2) Application management: Based on the above-mentioned intermediate results, the aggregation index interfaces of different dimensions are integrated to provide services for provincial localization construction. This module manages and schedules the application services running in the edge layer in a unified way, supports one-click installation and deployment, and dynamically schedules related computing and storage resources.

With the continuous development of AI and IoT technologies, meteorological observation stations have been equipped with high-definition cameras for live monitoring. Accordingly, a large number of video data and video transcoding requests are generated in the process of observation. With the disadvantages of insufficient computing resources and high latency, the traditional cloud computing architecture can hardly meet the demand of future meteorological services. By contrast, there are edge computing nodes between the cloud and terminal devices in edge computing, which can reduce interaction latency, raise the computing speed, and relieve the pressure of national data centers.

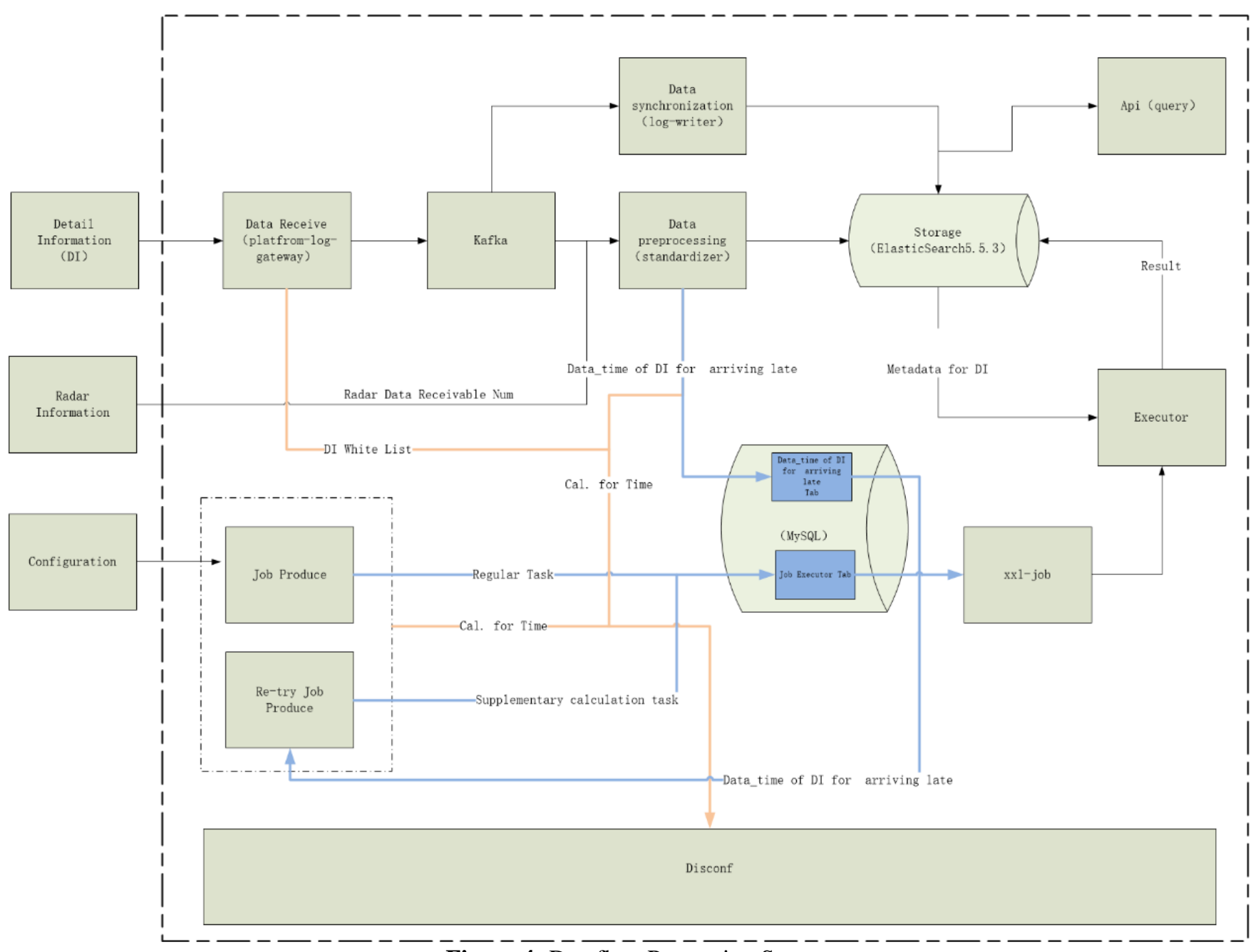

Figure 4: Dataflow Processing Structure

\section{Conclusion}

This paper introduces the development history of edge computing and analyzes two popular edge computing architectures: EdgeX Foundry and Baetyl2.0. With the feature of data processing and storage at the edge of network, edge computing meets the requirements of many applications for latency, data size, privacy and security. As a new computing paradigm, edge computing has attracted extensive attention from academia, industry and government. Finally, this paper 
summarizes the application characteristics of edge computing in meteorology, and proposes a architecture of information system based on the application background of meteorological observation. At present, the research and application of edge computing in meteorological application scenarios are still facing many challenges [15], such as bandwidth adaptation, minute-level meteorological data processing, security authentication and resource coordination, which are the main research directions in the future.

\section{References}

[1] Shi WS, Sun H, Cao J, et al. Edge Computing: A New Computing Model in the Age of Internet of Everything[J]. Journal of Computer Research and Development, 2017, 54 (5): 907-924.

[2] Lu HZ, Chen D, Fan B, et al. Progress and Case Study of Edge Computing Standardization[J]. Journal of Computer Research and Development, 2018, 55 (3): 487-511.

[3] Li LZ, Zhou PL, Cheng P \& Shi ZG. Architecture, Challenges and Applications of Edge Computing[J]. Big Data, 2019 (010):1-14.

[4] Shi WS, Zhang XZ, Wang YF, et al. Edge Computing: Present Situation and Outlook[J]. Journal of Computer Research and Development, 2019 (1): 1-21.

[5] EdgeX Foundry. EdgeX Foundry[OL]. https://wiki.edgexfoundry.org/display/FA/EdgeX+Foun dry+Project+Wiki

[6] Baetyl. Baetyl v2[OL]. https://docs.baetyl.io/en/latest/overview/architecture.ht ml\#edge-computing-framework

[7] Wang XF \& Han YW. Edge Intelligence Computing and Intelligent Edge Computing[J]. Zhangjiang Technology Review, 2019 (02): 10-12.

[8] Wang Z. Development Status and Prospect of Intelligent Edge Computing[J]. Artificial Intelligence, 2019 (05): 18-25.

[9] Quigley M, Conley K, Gerkey B, et al. ROS: An open-source robot operating system[EB/OL]. [2018-11-26].

http://www.will1owgarage.com/sites/default/files/icrao ss09-ROS.pdf

[10] Yi S, Qin Z, Li Q. Security and privacy issues of fog computing: A survey[C]//Proc of Int Conf on Wireless Algorithms, Systems, and Applications. Cham, Switzerland: Springer,2015:685-695

[11] SHI W, DUSTDAR S. The promise of edge computing[J]. Computer, 2016, 49(5): 78-81.

[12] CHI J, OWUSU E, YIN X, et al. Privacy partition: a privacy-preserving framework for deep neural networks in edge networks[C]//2018 IEEE/ACM Symposium on Edge Computing (SEC), February 24-25, 2018, Dubai, United Arab Emirates. Piscataway: IEEE Press, 2018: 378-380.

[13] Shen WH, He WC \& Sun C. Data Work of Meteorological Information Services from the Perspectives of Two Typical Applications[J]. iChina, 2017, (09): 71-76.

[14] Sun C, Xiao WM, Chen YT, Zeng L \& Zhang XY. Design of Real-Time Monitoring System for Integrated Meteorological Services[J]. Advances in Meteorological Science and Technology, 2018, (1):153-157.
[15] Shen WH. The value of meteorological big data lies in application[J]. iChina, Issue 7 of 2018. 